\title{
BIOMATERIALS BASED ON NANOHYDROXYAPATITE
}

\author{
Gabriela Ciobanu $^{\mathrm{a}^{*}}$, Constantin Luca ${ }^{\mathrm{a}}$, Octavian Ciobanu ${ }^{\mathrm{b}}$ \\ a"Gheorghe Asachi" Technical University of Iasi, Faculty of Chemical Engineering and Environmental Protection, \\ 63, Prof. dr. docent Dimitrie Mangeron Rd., Iasi 700050, Romania \\ b "Grigore T. Popa” University of Medicine and Pharmacy, Faculty of Medical Bioengineering, \\ 16, Universitatii str., Iasi 700115, Romania \\ *email: gciobanu03@yahoo.co.uk; phone(+40 741)02 51 63; fax (+40 232) 271311
}

\begin{abstract}
In this study, the porous hydroxyapatite-filled cellulose acetate scaffolds were prepared via dry-wet phase inversion method by dispersing hydroxyapatite nanoparticles in the polymeric matrix. The calcined hydroxyapatite prepared by wet precipitation method has the crystal size smaller than $50 \mathrm{~nm}$. The unfilled and hydroxyapatite-filled cellulose acetate scaffolds have an asymmetric structure consisting of two layers, the dense top layer (active layer) supported by the porous sub-layer (substructure). The cross-sectional SEM images revealed that hydroxyapatite nanoparticles were well dispersed in the cellulose acetate matrix.
\end{abstract}

Keywords: hydroxyapatite, cellulose acetate, scaffold.

\section{Introduction}

In the biological hard tissues the calcium phosphates are the most important inorganic constituents. From the calcium phosphate family, the hydroxyapatite $\mathrm{Ca}_{10}\left(\mathrm{PO}_{4}\right)_{6}(\mathrm{OH})_{2}$ is an important representative being present in bone, teeth, and tendons to give these organs stability, hardness and function [1]. The hydroxyapatite has many practical applications in the medicine and chemistry fields. In biomedical applications, the hydroxyapatite has been used as bioceramics due to its excellent biocompatibility and osteoconductivity properties [2]. The hydroxyapatite provides bioactivity but is brittle, particularly in highly porous scaffolds which may also exhibit low strength [3]. Therefore, dense or porous polymer-hydroxyapatite composites have been investigated for synthetic bone graft substitutes and bone tissue engineering scaffolds [4,5]. Recently, many research efforts are focused on combining the characteristics of both hydroxyapatite and polymers to create hybrid materials to be used for hard tissue replacement applications [6]. The polymer/hydroxyapatite composites have attractive features as candidates for novel bone substitutes because they may show bone-bonding capacity and mechanical performances derived from the organic substrate.

In this work, hydroxyapatite and cellulose acetate are combined to form hydroxyapatite-filled cellulose acetate scaffolds with applications in the biomedical field. The hydroxyapatite nanocrystals were dispersed within the cellulose acetate matrix for improving their properties. The effects of hydroxyapatite loading on the morphology and properties of the resultant scaffolds were investigated.

\section{Experimental}

The hydroxyapatite nanopowder was synthesized by wet chemical precipitation method, as described elsewhere [7]. Calcium nitrate $\mathrm{Ca}\left(\mathrm{NO}_{3}\right)_{2} \cdot 4 \mathrm{H}_{2} \mathrm{O}$, ammonium phosphate dibasic $\left(\mathrm{NH}_{4}\right)_{2} \mathrm{HPO}_{4}, \mathrm{HNO}_{3}$ and $\mathrm{NaOH}$ were purchased by Sigma-Aldrich (Germany). All reagents were of analytical grade and were used as received without further purification. Experiments were performed in distilled and deionized water. The $\mathrm{Ca}\left(\mathrm{NO}_{3}\right)_{2} \cdot 4 \mathrm{H}_{2} \mathrm{O}$ and $\left(\mathrm{NH}_{4}\right)_{2} \mathrm{HPO}_{4}$ were used as calcium source and as phosphorous source, respectively. An aqueous solution of $250 \mathrm{~mL}$ of $\mathrm{Ca}\left(\mathrm{NO}_{3}\right)_{2} \cdot 4 \mathrm{H}_{2} \mathrm{O}(0.01 \mathrm{M})$ was added drop-wise to an appropriate amount of $\left(\mathrm{NH}_{4}\right)_{2} \mathrm{HPO}_{4}(0.05 \mathrm{M})$ aqueous solution to achieve predetermined Ca/P atomic ratio of 1.67 . The suspension was adjusted to $\mathrm{pH}=11$ by adding small portions of $1 \mathrm{M} \mathrm{NaOH}$ and matured for 3 $\mathrm{h}$ at approximately $70{ }^{\circ} \mathrm{C}$ under magnetic stirring. The powder obtained was removed from the solution, washed with deionized water, dried at $110^{\circ} \mathrm{C}$ for $24 \mathrm{~h}$ and calcined at a temperature of $800^{\circ} \mathrm{C}$ during $3 \mathrm{~h}$. The phase composition of the hydroxyapatite powder was characterized by X-ray diffraction (XRD) with X'PERT PRO MRD diffractometer (PANalytical, Netherlands) using monochromatic CuKa radiation $(\lambda=0.15418 \mathrm{~nm}$ ), operating at $40 \mathrm{kV}$ and $50 \mathrm{~mA}$ over a $2 \theta$ range from $2^{\circ}$ to $70^{\circ}$. The average crystallite size $(D)$ of the hydroxyapatite powder was calculated from XRD data by the Scherrer equation, using the peak at $2 \theta=25.9^{\circ}$ for $(002)$ reflection. The morphology and chemical composition of the sample were studied by scanning electron microscopy (SEM) coupled with energy dispersive X-ray spectroscopy (EDX) with QUANTA 200 3D microscope (FEI, Netherlands). Silver sputtering was used to make the coating surfaces conductive for the SEM investigations. The $\mathrm{pH}$ measurements were realized with a Multi-Parameter Consort C831 (CONSORT, Belgium).

The pure cellulose acetate scaffold (denoted CA-HA-0) was prepared by the phase inversion method using cellulose acetate (CA) polymer and acetone as solvent, as described elsewhere [8]. The CA polymer and acetone were purchased by Sigma-Aldrich (Germany). The CA solution was made by dissolving a suitable quantity of CA polymer (20 wt.\%) in acetone at atmospheric pressure and $22^{\circ} \mathrm{C}$ for $3 \mathrm{~h}$. Then, the polymer solution was casted on a glass plate at 
a designated wet thickness using a casting knife at ambient temperature and left subsequently for 15 min in order to form the skin layer. After that, the cast film was dried at $70{ }^{\circ} \mathrm{C}$ for $24 \mathrm{~h}$ to remove residual solvents. The composite scaffolds were obtained by adding a calculated amount of HA into the CA polymer solution and mixing thoroughly before casting. The weight percentages of HA loaded in each scaffold were varied as stipulated in Table 1 . The casting and curing of the composite samples are identical with those of the pure CA scaffolds.

The characteristics of the unfilled and hydroxyapatite-filled cellulose acetate scaffolds.

\begin{tabular}{|c|c|c|c|c|c|c|}
\hline Sample & HA content $(\%)$ & $\begin{array}{l}\text { Thickness }^{\mathrm{a}} \\
(\mu \mathrm{m})\end{array}$ & $\begin{array}{l}\text { Pore diameter } \\
\text { in active layer } \\
(\mu \mathrm{m})\end{array}$ & $\begin{array}{c}\text { Pore diameter in } \\
\text { substructure }^{\mathrm{a}}(\mu \mathrm{m})\end{array}$ & $\begin{array}{l}\text { Porosity } \\
\quad(\%)\end{array}$ & $\begin{array}{l}\text { Density } \\
\left(\mathrm{g} / \mathrm{cm}^{3}\right)\end{array}$ \\
\hline CA-HA-0 & 0 & 208 & 1.33 & 99.3 & 69.7 & 0.209 \\
\hline CA-HA-10 & 10 & 178 & 1.08 & 70.1 & 65.2 & 0.314 \\
\hline CA-HA-20 & 20 & 127 & 0.97 & 42.7 & 51.8 & 0.327 \\
\hline CA-HA-30 & 30 & 86 & 0.94 & 20.9 & 40.1 & 0.342 \\
\hline
\end{tabular}

a) by SEM method;

b) by Bubble-point method.

The morphology and chemical composition of the samples were elucidated by scanning electron microscopy (SEM) coupled with energy dispersive X-ray spectroscopy (EDX), with QUANTA 200 3D Dual Beam scanning electron microscope (FEI Co., USA). For the SEM-EDX investigations, gold sputtering was used to create a conductive coating surface. The pore diameters have been determined by Bubble-point method with a laboratory instrument. The porosity $(\varepsilon)$ of the scaffolds was estimated by gravimetric method by the weight of liquid (isopropanol) contained in the scaffold pores, using the following equation:

$\varepsilon=\frac{w_{\mathrm{w}}-w_{\mathrm{d}}}{\rho_{\mathrm{w}} \cdot A \cdot L} \cdot 100$

where $w_{d}$ is the weight of the dry sample $(\mathrm{g}), w_{\mathrm{w}}$ is the weight of the wet sample after dipping into isopropanol for $2 \mathrm{~h}$ $(\mathrm{g}), \rho_{\mathrm{w}}$ is the isopropanol density $\left(0.785 \mathrm{~g} / \mathrm{cm}^{3}\right)$ at room temperature, $A$ is the effective area of the sample $\left(\mathrm{m}^{2}\right)$ and $L$ is the sample thickness $(\mathrm{m})$.

The feature characteristics of the prepared scaffolds are summarized in Table 1.

\section{Results and discussion}

The XRD method was applied to characterize the phase structure and crystallite size of the hydroxyapatite sample. The wide-angle XRD pattern (Figure 1a) indicate that the calcined hydroxyapatite powder has the characteristic peaks in the $2 \theta$ regions of $21^{\circ}-29^{\circ}, 32^{\circ}-34^{\circ}, 39^{\circ}-41^{\circ}, 46^{\circ}-54^{\circ}$, in good agreement with the hexagonal hydroxyapatite phase (JCPDS Data Card 09-0432). The average crystallite size $(D)$ of the hydroxyapatite powder was calculated from XRD data using the Scherrer equation, being of $49 \mathrm{~nm}$.

The SEM and EDX methods were performed in order to determine the morphology and surface elemental composition of the hydroxyapatite. Figure 1b shows the SEM micrographs of the calcined sample. It is seen that the hydroxyapatite powder is composed of nanosized primary particles which tends to form agglomerates with intergranular micropores. The EDX analysis (Figure 1c) of the sample confirms the presence of the Ca, $\mathrm{P}$, and $\mathrm{O}$ elements in HA crystallites. The Ca/P mole ratio was of 1.675 mostly close to 1.67 corresponding to the stoichiometric hydroxyapatite [1].

The formation of CA asymmetric scaffold is the result of microphase separation phenomena that occur during an evaporation step and/or quench step of an initially thermodynamically stable polymer solution. Consequently, the scaffold structure is affected by the conditions of scaffold preparation [9-11].

The unfilled and hydroxyapatite-filled cellulose acetate scaffolds with an asymmetric and interconnected porous structure were obtained by applying a phase inversion process. These asymmetric scaffolds have a very thin skin layer supported by a more open porous substructure, as seen in Figure 2. The formation of skin layer was attributed to the solvent evaporation during the microphase separation process. The size of the pores in the skin layer is very small compared to that in the substructure (Table 1). The CA scaffolds have internal micropores and macropores. At external surface of the scaffold, on the skin layer, many nanopores were formed during phase inversion process. 

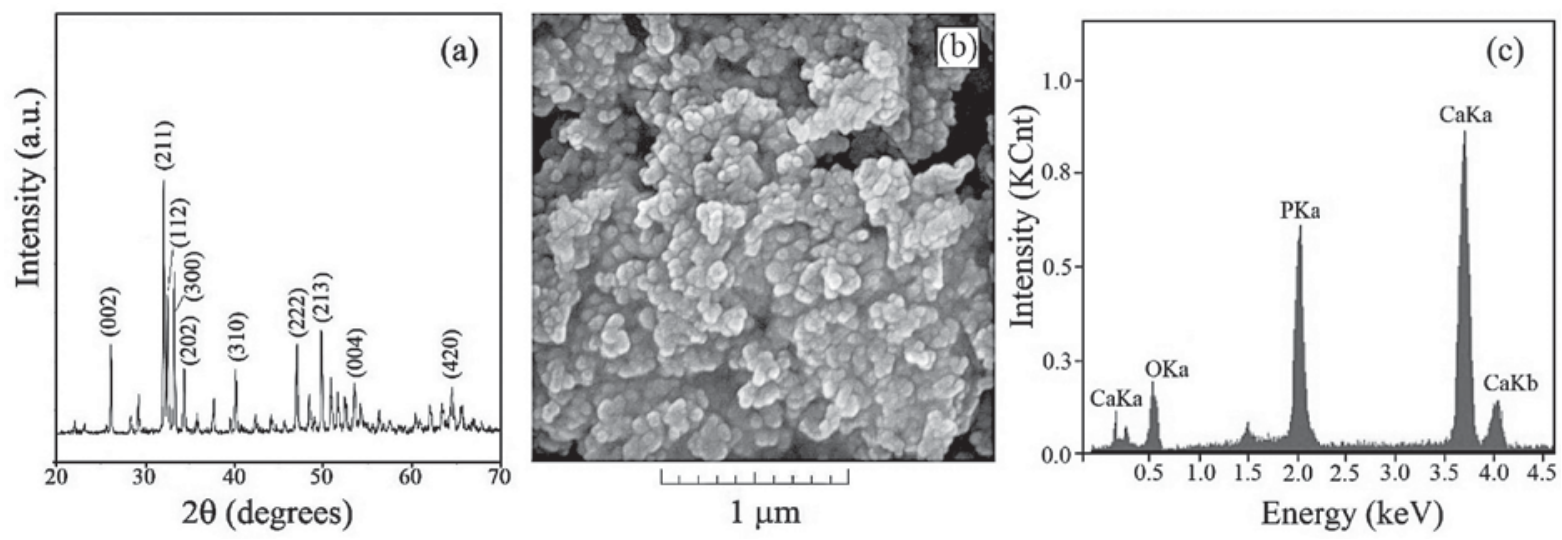

Figure 1. XRD pattern (a), SEM image (b) and EDX spectrum (c) of the hydroxyapatite sample.

The porosity of the scaffolds is high, and therefore this porous structure provides a good microenvironment for cell adherence, growth and proliferation. As shown in Table 1, the porosity of the composite samples decreases with the addition of the hydroxyapatite. The SEM images of the scaffolds in the cross-section (Figure 2) confirm the good incorporation of the hydroxyapatite in the polymeric matrix. Hydroxyapatite crystals can be seen spread over the internal surface of the scaffold as white spots. The most hydroxyapatite particles were dispersed uniformly within the scaffolds substructure, excepting for very few clusters that might have been resulting from nanosized particles coalescing in the polymeric matrix. We assume that the occurrence of these clusters is due to the fact that the surface of the hydroxyapatite contains $\mathrm{OH}$ functionalities (hydrophilic $\mathrm{P}-\mathrm{OH}$ groups) causing inherent hydrophilicity to these particles. Therefore, some hydroxyapatite nanoparticles tend to adhere to each other via hydrogen bonding forming some irregular agglomerations within scaffold. This could reduce the efficiency of the dispersion process especially in the samples with higher hydroxyapatite particle contents $(>30 \%)$.
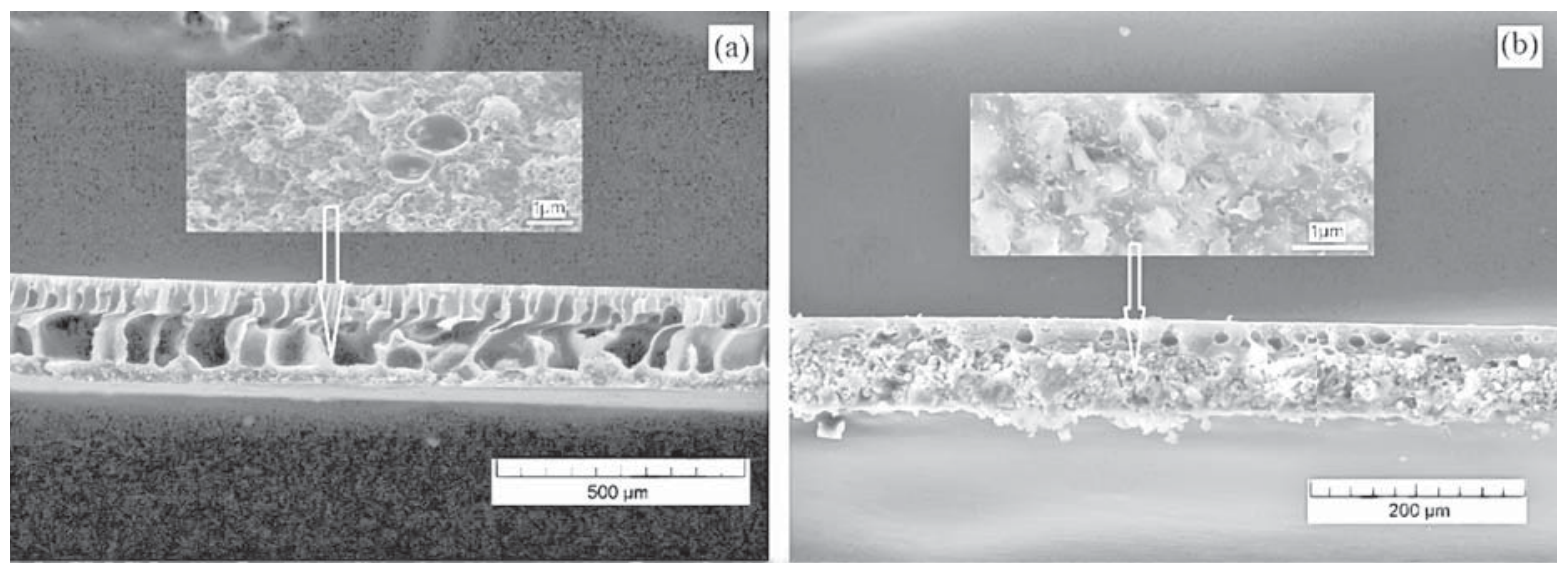

Figure 2. The SEM images of the scaffolds in the cross-section: a) CA-HA-0 and b) CA-HA-30 samples.

The incorporation of the hydroxyapatite crystals in CA matrix induces some changes into scaffold morphology. A good dispersion of the hydroxyapatite is important but difficult to obtain especially when small particles of hydroxyapatite are used. Interactions exist between hydroxyapatite and the used polymer. The hydroxyapatite acted as a cross-linker on the CA polymer and, finally resulted material being a reinforced scaffold.

The brittleness of the final scaffolds was influenced by the amount of hydroxyapatite in the scaffold. When the amount of hydroxyapatite particles in the composites was less than $30 \%$, the obtained scaffolds were intact and strong. On the other hand, when the amount of hydroxyapatite was higher than $30 \%$, the scaffolds possessed a relatively coarse surface becoming brittle.

Finally, the results of the present study indicate that the asymmetric hydroxyapatite-filled cellulose acetate scaffolds could be obtained by phase inversion method. 


\section{Conclusions}

The hydroxyapatite nanopowder was produced by coprecipitation reactions. The XRD pattern of the hydroxyapatite sample display well-defined and sharp peaks in agreement with a high degree of crystallinity. The porous hydroxyapatite-filled cellulose acetate scaffolds were prepared via dry-wet phase inversion method. The unfilled and hydroxyapatite-filled cellulose acetate scaffolds have an asymmetric structure consisting of two layers: the dense top layer supported by the porous sub-layer. The SEM-EDX studies of the composites confirm the presence of the hydroxyapatite nanocrystals in the cellulose acetate matrix. The most hydroxyapatite particles were dispersed uniformly within the scaffolds substructure, excepting for very few clusters that might have been resulting from nanosized particles coalescing in the cellulose acetate matrix. The porosity of the scaffolds is high, and therefore this porous structure provides a good microenvironment for cell adherence, growth and proliferation.

\section{References}

1. Dorozhkin, S.; Epple, M. Biological and Medical Significance of Calcium Phosphates. Angewandte Chemie International Edition, 2002, 41, pp. 3130-3146.

2. Kong, L.; Gao, Y.; Lu, G.; Gong, Y.; Zhao, N.; Zhang, X. A study on the bioactivity of chitosan/nanohydroxyapatite composite scaffolds for bone tissue engineering. European Polymer Journal, 2006, 42, pp. 3171-3179.

3. Kane, R.; Roeder, R. Effects of hydroxyapatite reinforcement on the architecture and mechanical properties of freeze-dried collagen scaffolds. Journal of the Mechanical Behavior of Biomedical Materials, 2012, 7, pp. 41-49.

4. El-Kady, A.M.; Mohamed, K.R.; El-Bassyouni, G.T. Fabrication, characterization and bioactivity evaluation of calcium pyrophosphate/polymeric biocomposites. Ceramics International, 2009, 35, pp. 2933-2942.

5. Habraken, W.J.; Wolke, J.G.; Jansen, J.A. Ceramic composites as matrices and scaffolds for drug delivery in tissue engineering. Advanced Drug Delivery Reviews, 2007, 59, pp. 234-248.

6. Yarlagadda, P.; Chandrasekharan, M.; Shyan, J.Y.M. Recent advances and current developments in tissue scaffolding. Bio-Medical Materials and Engineering, 2005, 15, pp. 159-177.

7. Ciobanu, G.; Ilisei, S.; Harja, M.; Luca, C. Removal of Reactive Blue 204 dye from aqueous solutions by adsorption onto nanohydroxyapatite. Science of Advanced Materials, 2013, 5, pp. 1090-1096.

8. Ciobanu, G.; Carja, G.; Ciobanu, O. Preparation and characterization of polymer-zeolite nanocomposite membranes. Materials Science and Engineering C - Materials for Biological Applications, 2007, 27, pp. 1138-1140.

9. Ciobanu, G.; Carja, G.; Ciobanu, O. Use of SAPO-5 zeolite-filled polyurethane membranes in wastewater treatment. Desalination, 2008, 222, pp. 197-201.

10. Hou, Q.P.; Grijpma, D.W.; Feijen, J. Porous polymeric structures for tissue engineering prepared by a coagulation, compression moulding and salt leaching technique. Biomaterials, 2003, 24, pp. 1937-1947.

11. Guan, J.; Fujimoto, K.; Sacks, M.; Wagner, W. Preparation and characterization of highly porous, biodegradable polyurethane scaffolds for soft tissue applications. Biomaterials, 2005, 26, pp. 3961-3971. 